\section{Lebanon: Encouraging Signs}

The Rammal Medal is awarded each year to an eminent physicist from a country surrounding the Mediterranean who has given a modern perspective to the region's long tradition of scientific exchange. It was won this year by Celal Sengör, a geologist from Istanbul whose work has gained international recognition in throwing new light onto our conception of how the western part of Asia was formed geologically. He is a member of several academies including the Academia Europea and the new Academy of Turkey. The award ceremony took place in March 1995 on a campus of the Lebanese University, with the Minister of Culture and Higher Education representing the President of the Lebanese Republic. It had sufficient impact to be reported on in the Beruit press.

For someone visiting Lebanon for the first time, it was moving to see the destruction caused by a devastating war as well as the encouraging signs of a new start. Talking to people in the street and elsewhere, it was hard to believe that the fighting had been fierce only four years before.

The country has no less than 17 universities, the most well-known being the Lebanese University, the English speaking University, the French speaking St. Joseph University, and the Arab University. Visits convinced the European delegation to the award ceremony of the determination of Lebanese scientists to resume scientific activities. Much effort was put on teaching as well as on research at an international level. Projects were flourishing everywhere, even if their realisation was impeded by difficult material conditions. For instance, the electricity supply was not yet operating 24 hours a day, although there was a good hope that the situation would improve.

France's Conseil National de la Recherche Scientifique (CNRS) is presently promoting research projects in the Lebanese University and in other institutions. The danger of scientific isolation is nevertheless serious: e-mail is just starting to become available (with CNRS help), and most subscriptions to scientific journals were cancelled long ago. In addition, many journal collections have been destroyed by the war. Even complimentary publications such as Laser Focus and Lasers and Optronics, while easy to obtain in Europe, are not received although they contain much technical information which could be useful for applied projects. It is clear that Lebanese colleagues need our help if they are to achieve a satisfactory level of scientific communication and exchange.

F. Laloë, ENS, Paris

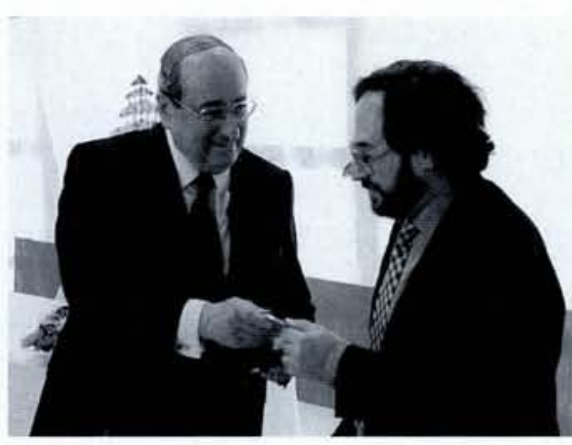

C. Sengör, on the right, receiving the $1995 \mathrm{Hammal}$ Medal from Lebanon's Minister of Culture and Higher Education.

\title{
Middle East: A First Step
}

Many appreciate that physics can act as a precious bridge across political and ideological barriers and all the misunderstanding which usually goes with them. Such was the case between east and west at the peak of the cold war, when EPS played an important role in this respect. Other regions can also benefit much from contacts developed around scientific activities. This is especially true in the Middle East where conditions are ripening but where the peace process still needs all the help it can get.
The Sinaï Meeting on High-Energy Physics, Condensed Matter and Environmental Physics (Dahab, Egypt; 1926 November 1995) represented a first step, let us hope, for increasing scientific cooperation throughout the Middle East. It was the first physics meeting to bring together Egyptian, Israeli, Jordanian, and Palestinian physicists. There were altogether about 1so participants: half were students, and all national groups were well represented. In particular, it was the first meeting at which Palestinian physicists were an identifiable entity. Senior participants from Europe, the USA and Morocco contributed much to the success of the enterprise, and everyone felt that they had spent a fruitful week listening to excellent talks, discussing physics and learning much about the ways of the others. Fears and difficulties were overcome and a collaborative spirit prevailed.

The meeting was made possible thanks to the enthusiasm of Sergio Fubini (Turin and CERN) and the indefatigable Alberto Devoto (Cagliari). Equally important were the encouragement and goodwill of science authorities in Egypt, Israel and Jordan and of representatives of the Palestinians. Most of the funding was provided by the ICTP (Trieste) and other Italian sources, with CERN and UNESCO as sponsors. Israel made a sizable contribution, and Eliezer Rabinovici took an active part in the organization. This may herald the special role which Israel can eventually play as a hub for scientific activities once the peace process has progressed.

The conference focused on fundamental and topical questions in particle and condensed matter physics. There was a strong theoretical slant that reflected the organizers' main interests and the fact that theoretical aspects of the environment and superconductivity were considered at length.

A distinguished panel including the Egyptian Minister for Research, the President of the Israeli Academy of Sciences and the Scientific Director of UNESCO (who happens to be Jordanian) formally opened the meeting. The Italian Minister of Research could not attend at the last minute but sent a warm letter which was read out by S. Fubini. There followed a round-table discussion with many participants expressing their views. Harry Lipkin (Wiezmann Institute) expressed his heartfelt wish that participants would learn much not only about physics but also about how others think, thus dispelling the considerable misunderstanding that exists. The meeting undoubtedly achieved these aims. The participants felt that they had gone through a wonderful experience which would have been impossible a year ago.

The road to extensive scientific cooperation in the Middle East remains long and treacherous, and the pace will be different in different countries. Yet a purposeful step forward has now been made. The success of this first meeting calls for others. Two that focus on particular issues are already at the planning stage.

M. Jacob, CERN 